\title{
Primeros registros de helmintos parásitos en Fulica ardesiaca (Aves: Rallidae) para el Perú : Pantanos de Villa - Lima
}

\section{First record of helminth parasites in Fulica ardesiaca (Aves: Rallidae) from Peru: Pantanos de Villa - Lima}

\author{
Gisella Guillén ${ }^{1}$ y Elizabeth Morales ${ }^{1,2}$
}

Presentado: $13 / 11 / 2003$

Aceptado: 28/11/2003

\section{RESUMEN}

En el presente trabajo se registran por primera vez para el Perú, los parásitos de un ejemplar de Fulica ardesiaca «gallareta andina» colectada en los Pantanos de Villa. Se colectaron un total de 698 especimenes: los tremátodos Notocotylus pacifera y Psilostomum sp. con 529 y 149 individuos respectivamente y el céstodo Diorchis americana, con 20. El presente trabajo constituye el primer reporte para el Perú de helmintos en $F$. ardesiaca y Psilostomum sp. y $D$. americana son primeros registros para la helmintofauna peruana.

Palabras claves: Fulica ardesiaca, Pantanos de Villa, parásitos, Notocotylus, Psilostomum, Diorchis, Lima, Perú.

\section{ABSTRACT}

Two trematodes and one cestode of Fulica ardesiaca from Pantanos de Villa, Lima, Peru are registered at first time. Total of 698 parasites were counted, 529 individuals of Notocotylus pacifera, 149 individuals of Psilostomum sp. and 20 individuals of Diorchis Americana. This work is the first report in F. ardesiaca from Peru; while Psilostomum sp. and D. americana are first registrations for the Peruvian's helminthes.

Keywords: Fulica ardesiaca, Pantanos de Villa, parasites, Notocotylus, Psilostomum, Diorchis, Lima, Peru.

\section{Introducción}

Los «Pantanos de Villa», son humedales considerados como la única zona de vida silvestre en la cuidad de Lima, ubicado al sur de la ciudad, en el distrito de Chorrillos; posee un área total de 276 ha y un rango altitudinal que varía desde $1 \mathrm{~m}$ bajo el nivel del mar hasta 5 $\mathrm{m}$ sobre el mismo. Esta zona alberga una gran diversidad de aves (119 especies) entre residentes y migratorias (Pautrat y Riveros, 1998), asociadas a los diversos tipos de hábitat que encontramos a lo largo del pantano. Fulica ardesiaca , «gallareta andina», ave estudiada

\footnotetext{
${ }^{1}$ Laboratorio de Zoología de Invertebrados. Facultad de Ciencias Biológicas. UNMSM. Apartado 110058, Lima 11.Perú.

${ }^{2}$ Laboratorio de Helmintología. Museo de Historia Natural-UNMSM.

E-mail, Gisella Guillén: gikaguia@yahoo.com
}

en el presente trabajo, es una especie acuática, común y residente de los Pantanos de Villa, perteneciente al Orden Gruiformes, Familia Rallidae. Son aves regordetas, de coloración gris pizarra y de cabeza negra. Construyen sus nidos sobre la vegetación acuática cercana a la protección del totoral. Habita en lagos y lagunas andinas desde el sur de Colombia hasta el norte de Argentina, además de las costas peruanas. Su alimentación se basa en plantas acuáticas, granos y semillas, algunas veces se alimenta también de crustáceos, insectos y caracoles (Fjeldså y Krabbe, 1990). F. ardesiaca tiene como sinónimo a $F$. americana, y actualmente se le considera una subespecie, F. americana ardesiaca (Fjeldså y Krabbe, 1990). 
No existen antecedentes en el Perú de estudio de parásitos en esta especie, ni reportes en Sudamérica. Sin embargo, se han registrado helmintos de $F$. americana en Norteamérica y de F. atra en Europa. F. americana fue estudiada por Noble (1939) quien reportó un tremátodo identificado como $N$. pacifera.

\section{Material y métodos}

Se realizó la autopsia de un ave encontrada muerta y flotando en la orilla de la Laguna Mayor, en octubre de 1998. Las vísceras fueron preservadas en formol $10 \%$ y transportadas al Laboratorio de Zoología de Invertebrados de la Facultad de Ciencias Biológicas de la Universidad Nacional Mayor de San Marcos, donde se analizaron bajo el microscopio estereoscopio en busca de parásitos.

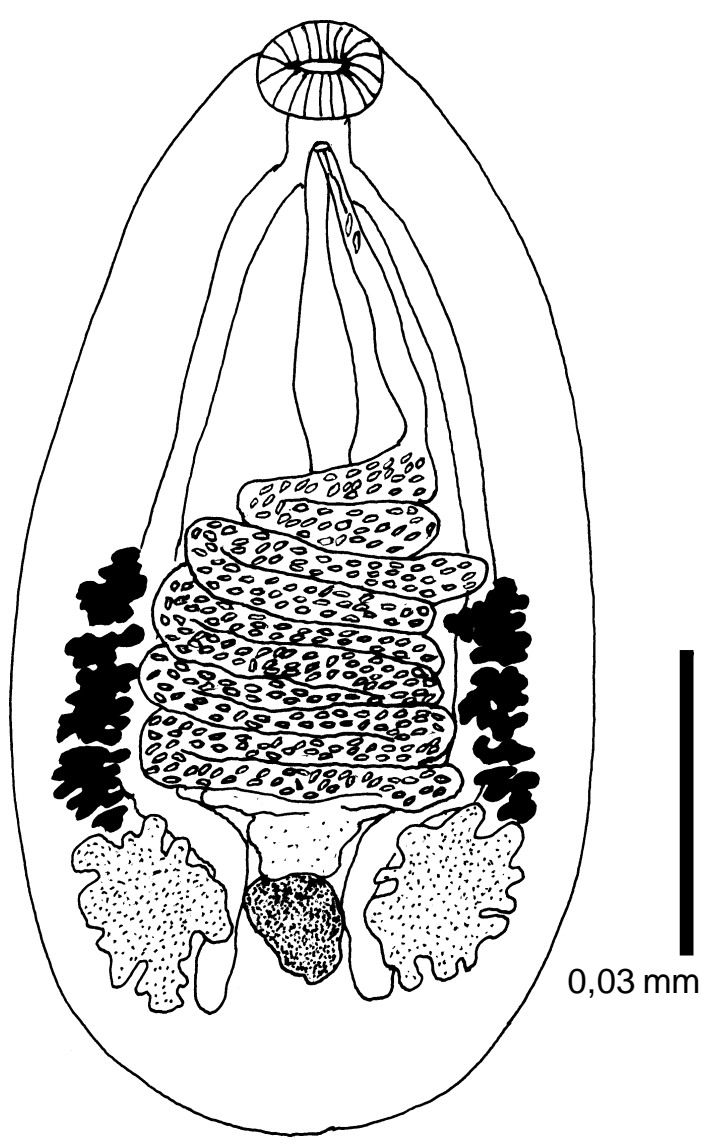

Figura 1. Notocotylus pacifera.
Los helmintos hallados fueron fijados en formol 5\% y posteriormente en alcohol $70 \%$. Algunos de los especimenes, se aplanaron y colorearon con Hematoxilina de Delafield o carmín de Semichon, siendo finalmente montadas en bálsamo de Canadá. Las medidas se tomaron con ayuda de un ocular micrométrico calibrado y los esquemas se realizaron utilizando una cámara lúcida Carl Zeiss. Algunas características de los parásitos se tomaron de muestras preservadas.

La identificación de los parásitos se hizo con ayuda de las claves taxonómicas de Dawes (1946) y Fish and Wildlife Service (1981) y las descripciones de Suresh (1952), Lumsden y Zischke (1962), Dollfus (1975), Tantaleán et al. (1974/1975, 1992).

\section{Resultados}

Se hallaron los siguientes helmintos parásitos: los tremátodos Notocotylus pacifera (Noble, 1933) Harwood, 1939 y Psilostomum sp. (Looss, 1899), el céstodo Diorchis americana (Ransom, 1909). Del total de 698 parásitos el 75,8\% (529) fueron $N$. pacifera, $21,8 \%$ (149) Psilostomum sp. y 2,9\% (20) D. americana.

En el contenido intestinal también se encontró restos de plantas y conchas de caracoles de la especie Melanoides tuberculata. Debido a que los gasterópodos son huéspedes intermediarios de digeneos pensamos que podrían constituirse en parte del ciclo de los digeneos hallados.

\section{Taxonomía y descripción}

\author{
Clase Trematoda Rudolphi, 1808 \\ Orden Notocotyliformes Travassos, 1950 \\ FAMilia Notocotylidae LuHe, 1909 \\ Subfamilia Notocotilynae Kossack, 1911
}

\section{Notocotylus pacifera (Noble, 1939) Harwood, 1939}

(Fig. 1)

Cuerpo ovalado, un poco alargado, sin espinas, carecen de ventosa ventral. Posee tres hileras de glándulas ventrales, las hileras late- 


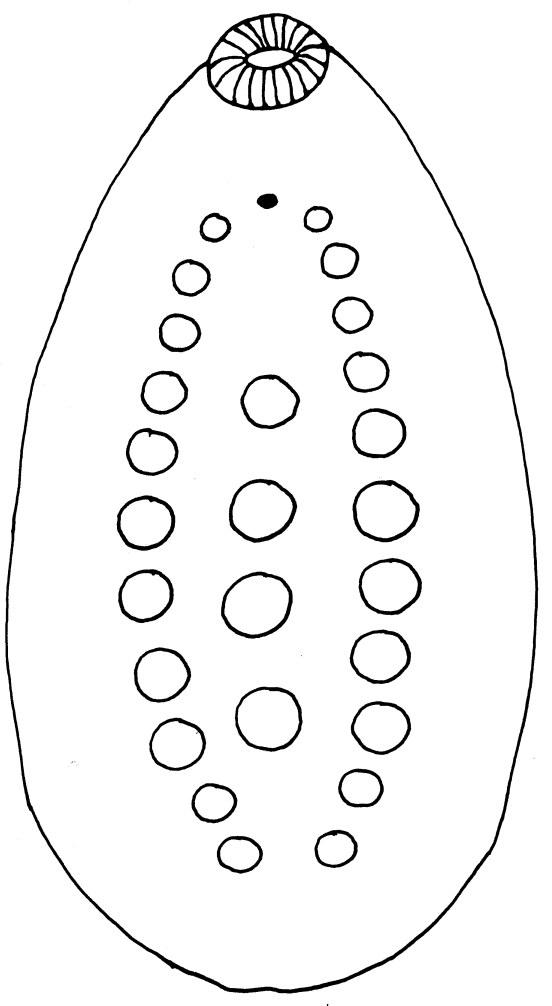

Figura 2. Notocotylus pacifera. Posición de las glándulas ventrales

rales con 9 a 11 glándulas cada una y la hilera del medio con 4 glándulas (Fig. 2).

El cuerpo mide de $0,62 \mathrm{~mm}$ a 2,1 $\mathrm{mm}$ de largo por $0,38 \mathrm{~mm}$ a $1,425 \mathrm{~mm}$ de ancho; la cutícula con un espesor promedio de 0,0085 $\mathrm{mm}$. La ventosa oral es terminal, midiendo de $0,077 \mathrm{~mm}$ a $0,102 \mathrm{~mm}$ de largo por $0,075 \mathrm{~mm}$ a $0,125 \mathrm{~mm}$ de ancho. La boca tiene una medida promedio de $0,052 \mathrm{~mm}$.

Los ciegos intestinales se bifurcan después del esófago. Poro genital cercano a la bifurcación. Los testículos son extracecales y posteriores, de forma lobulada con hendiduras y una medida promedio de $0,153 \mathrm{~mm}$ de largo por $0,095 \mathrm{~mm}$ de ancho. La bolsa del cirro desarrollada situada anteriormente, desemboca en el poro genital midiendo $0,223 \mathrm{~mm}$ de largo por $0,065 \mathrm{~mm}$ de ancho.

El ovario es intertesticular y de bordes en- teros, midiendo $0,1 \mathrm{~mm}$ de largo por $0,082 \mathrm{~mm}$ de ancho; útero dirigido anteriormente ocupando casi toda la parte media del individuo. Las glándulas vitelógenas son laterales extracecales, extendiéndose desde la zona ecuatorial del cuerpo hasta delante de los testículos, estos miden $0,032 \mathrm{~mm}$ de largo por $0,017 \mathrm{~mm}$ de ancho.

Los huevos son ovoides, de cáscara lisa y provistos en cada lado de un filamento que es varias veces más largo que el tamaño del huevo, con medida de $0,2 \mathrm{~mm}$ de largo por 0,0133 mm de ancho (Fig. 3).

Algunos individuos presentaron de 9 a 11 glándulas ventrales en las hileras laterales, difiriendo con los reportes dados por Tantaleán et al. (1974/1975) donde describe a $N$. pacifera con un número de 11 glándulas para cada hilera lateral, sin embargo en Fish and Wildlife Service (1981) lo describen con un número de 10 a 12 glándulas laterales y de 4 a 5 glándulas en la hilera media.

Dubois (1951) describe a $N$. pacifera como sinónimo de N. gibbus (Mehlis en Creplin, 1816), presentando un número de glándulas ventrales laterales de 10 a 11 y media de 4 a 5 y con una

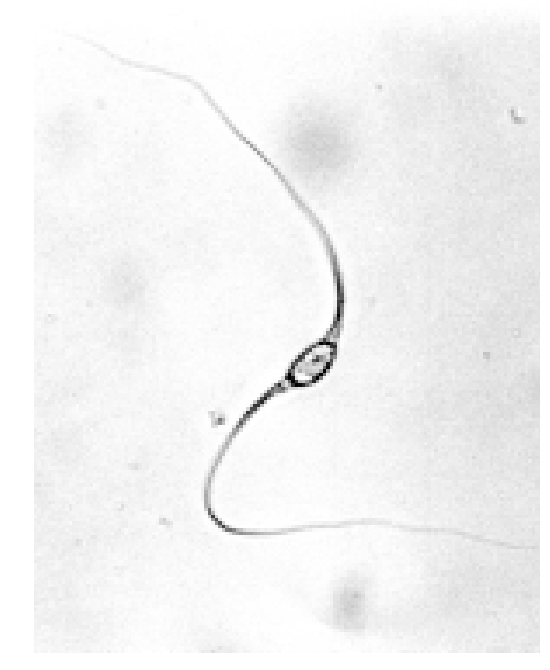

Figura 3. Notocotylus pacifera. Microfotografia del huevo. 
longitud del cuerpo entre $1-3,2 \mathrm{~mm}$ por $0,5-$ $1,4 \mathrm{~mm}$ de ancho, siendo reportado para $F$. atra, F. americana y Gallinula chloropus, procedentes de Europa, Japón, California y México.

N. pacifera ha sido estudiada por Tantaleán et al. (1974/ 1975, 1992) en el ciego de Cairina moschata «pato doméstico», procedente de Huancayo y Lima, por lo que nuestro hallazgo constituye el segundo registro de la especie en un nuevo huésped en el Perú.

\section{Clase Trematoda Rudolphi, 1808 \\ Orden $\quad$ Echinostomida LaRue, 1957 \\ FAMILIA PSILOSTOMATIDAE (ODHNER 1911) NICOLL, 1935}

\section{Psilostomum sp. (Looss, 1899)}

(Fig. 4)

Cuerpo alargado un poco aplanado, con acetábulo muy desarrollado y situado anteriormente. El cuerpo cuya parte anterior más ancha que la posterior mide de 1,35 a 2,33 mm de largo por 0,39 a $0,65 \mathrm{~mm}$ de ancho.

La cutícula tiene un espesor de $0,063 \mathrm{~mm}$ y posee pequeñas espinas. La ventosa oral es terminal y desarrollada, mide $0,245 \mathrm{~mm}$ de largo por $0,397 \mathrm{~mm}$ de ancho. El acetábulo 0,452 por $0,3975 \mathrm{~mm}$, posee una faringe desarrollada sin esófago; los ciegos intestinales se bifurcan anterior al acetábulo, siguiendo el largo del cuerpo.

Las gónadas son redondeadas y postacetabulares. El ovario cercano al acetábulo mide de $0,9 \mathrm{~mm}$ a $0,12 \mathrm{~mm}$ de largo por 0,12 a $0,152 \mathrm{~mm}$ de ancho. Las glándulas vitelógenas son foliculares y se sitúan lateralmente desde el extremo posterior del acetábulo hasta extremo posterior del cuerpo Los testículos son postováricos dispuestos uno detrás de otro y miden $0,198 \mathrm{~mm}$ por $0,166 \mathrm{~mm}$. El saco del cirro se curva alrededor del acetábulo y tiene una medida promedio de $0,187 \mathrm{~mm}$ de largo. El poro genital se sitúa delante del acetábulo.

Los huevos son grandes y poco numerosos, de cáscara lisa y delgada, miden 0,144

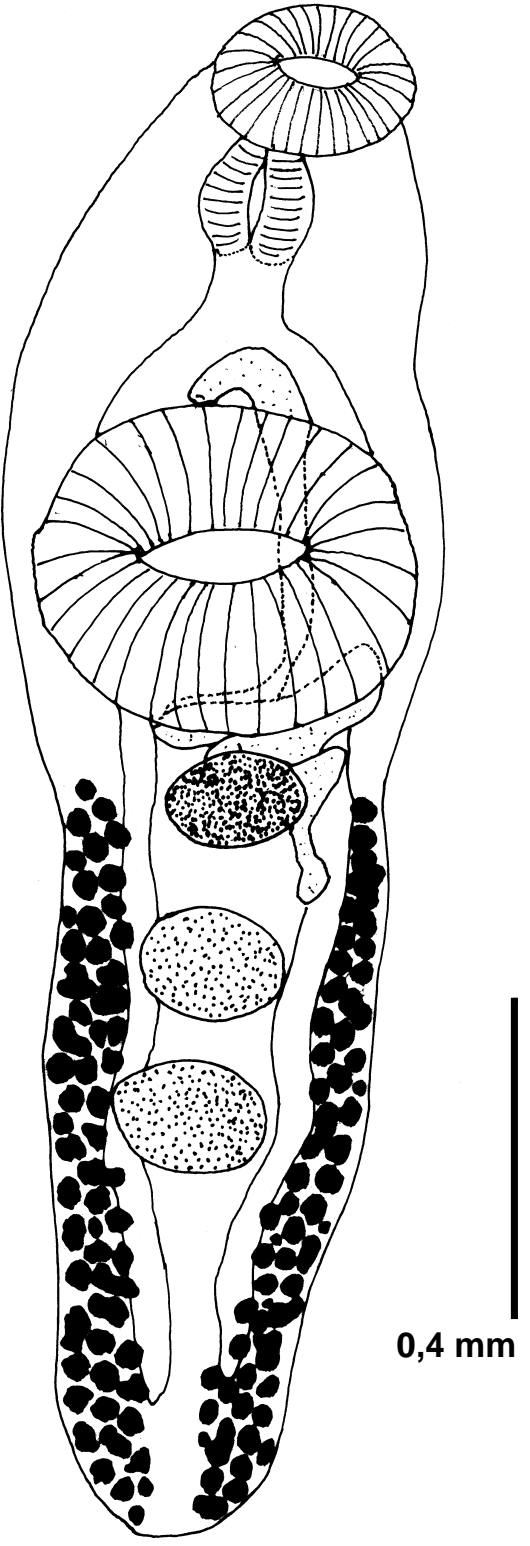

Figura 4. Psilostomun sp.

$\mathrm{mm}$ de largo por 0,088 $\mathrm{mm}$ de ancho. Los individuos observados en $F$. ardesiaca presentaron escaso número de huevos, en algunos casos solo tres huevos.

El género ha sido reportado en Asia, Norteamérica, Europa en el intestino de patos, cisnes, ostreros (Dawes, 1946 y Fish and Wildlife Life Service, 1981).

Este tremátodo constituye un primer registro para la helmintofauna peruana. 


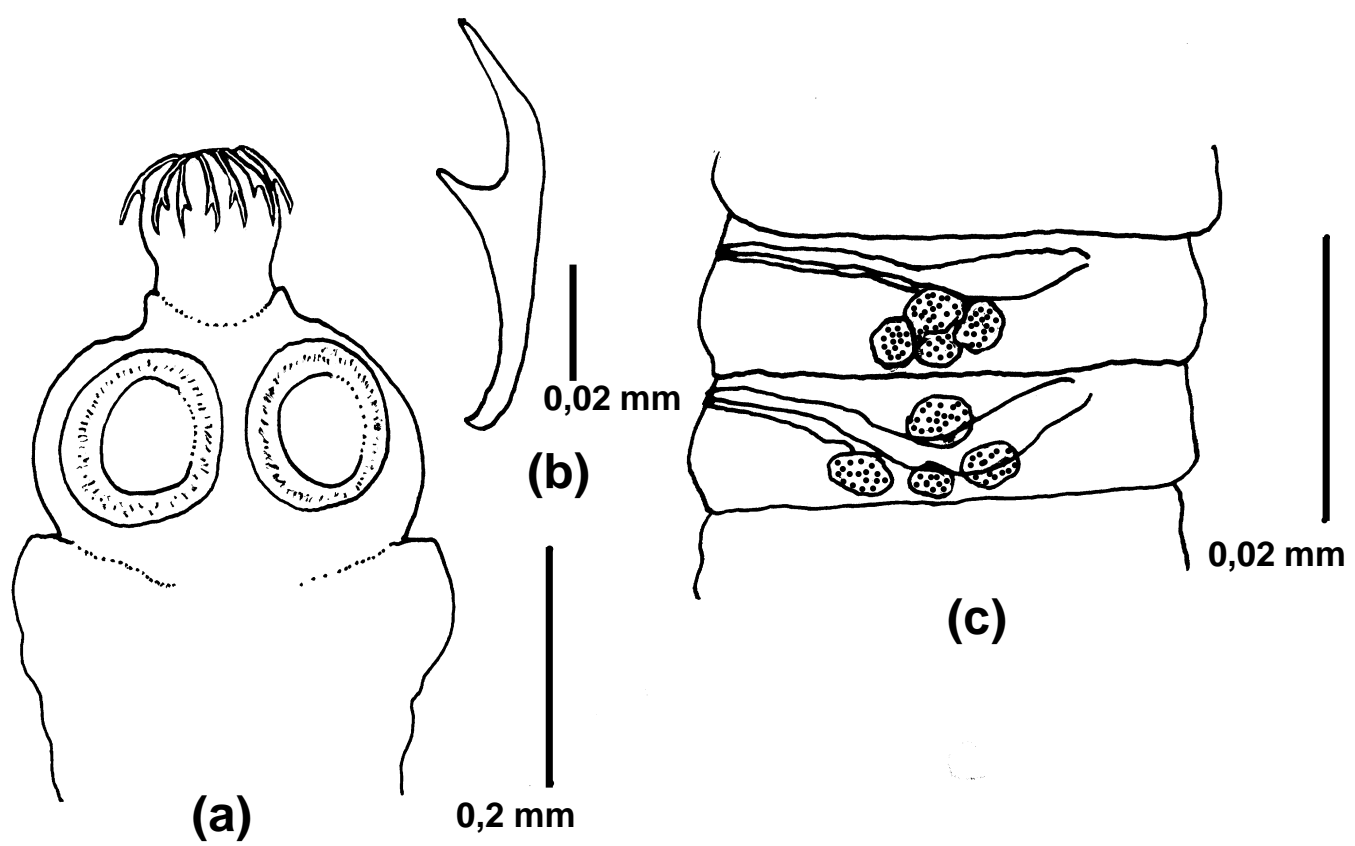

Figura 5. Diorchis americana. (a) Escólex, (b) gancho del escólex, (c) Proglótidos maduros

$\begin{array}{ll}\text { Clase } & \text { Cestoda Rudolphi, 1808 } \\ \text { Subclase } & \text { Cestodaria Monticelli, 1892 } \\ \text { Orden } & \text { Cyclophyllidea Braun, 1900 } \\ \text { Familia } & \text { Hymenolepidae Ariola } 1899\end{array}$

\section{Diorchis americana Ransom 1909}

(Fig. 5)

Los especímenes miden de $3,3 \mathrm{~mm} \mathrm{a} 6,8 \mathrm{~mm}$ de largo por 0,2 a $0,34 \mathrm{~mm}$ de ancho. El escólex posee rostelo armado con 10 ganchos formando una corona y mide 0,362 por $0,325 \mathrm{~mm}$, presenta cuatro ventosas, cada una de las cuales posee espinas pequeñas en su borde. Las ventosas miden de 0,11 a 0,137 mm de diámetro. Los ganchos miden en promedio $0,0725 \mathrm{~mm}$. El cuello está presente. Los proglótidos son más anchos que largos. El poro genital es unilateral. Los testículos lobulados en número de dos, con medida promedio de $0,05 \mathrm{~mm}$. Ovario lobulado, situado entre los testículos, con diámetro promedio de $0,025 \mathrm{~mm}$.

Suresh (1952), describe a D. americana, escólex que mide entre 0,3 y $0,32 \mathrm{~mm}$, con la presencia de 10 ganchos rostelares cada una de las cuales mide de 0,069 a $0,073 \mathrm{~mm}$ de longitud, siendo estas medidas muy cercanas a las observadas en nuestros especimenes.

D. americana fue descrita por primera vez por Ransom, 1909 en Fulica americana para Norteamérica (Dollfus, 1975); siendo posteriormente reportada para las especies Gallus gallus, Dendrociatta sp., Gallinula Chloropus y Fulica atra para Norteamérica, India y Europa (Schmidt, 1986).

Este céstodo también constituye un nuevo registro para el Perú. No se ha encontrado reportes para Sudamérica. Su hallazgo permite una ampliación en la distribución de la especie.

Debido a la carga parasitaria hallada se sugiere que podría tener repercusión en el bienestar de la población de Fulica ardesiaca, en los Pantanos de Villa.

\section{Agradecimientos}

Expresamos nuestro agradecimiento al $\mathrm{Sr}$. Eduardo Maguiña, miembro del Área Científica del Centro de Interpretación Pantanos de Villa del SERPAR-LIMA, por la donación del ave y al profesor Ruperto Severino, Jefe del Labora- 
torio de Zoología de Invertebrados de la Facultad de Ciencias Biológicas de la Universidad Nacional Mayor de San Marcos por las facilidades prestadas para esta investigación.

\section{Literatura citada}

Dawes, B. 1946. The Trematoda. Cambridge. London. $644 \mathrm{pp}$.

Dubois, G. 1951. Etude des trematodes NordAmericains de la collection E.L. Schiller et revisión du genre Notocotylus Diesing, 1839. Bull. Soc. Neuch. Sci. Nat. 74, 41 - 76.

Dollfus, R., Ph. 1975. Miscellanea Helminthologica Maroccana. Bull. Mus. Natn. Hist. Nat. Paris. $3^{\mathrm{e}}$ sér. Zoologie 212. $\mathrm{N}^{\circ}$ 302: 659 - 684.

Lumsden, R. and J.Zischke. 1962. Studies on Trematodes of Louisiana Birds. Z. Parasitenkunde 22, 316-366.
Fjeldså, J. and N. Krabbe. 1990. Bird of the High Andes. Zoological Museum. University of Copenhagen. 876 pp.

Fish and Wildlife Life Service. 1981. Key to Trematodes Reported in Waterfowl. Resource Publication 142.

Pautrat, Ly J. Riveros. 1998. Evaluación de la Avifauna de los Pantanos de Villa. En : Cano, A. y K. Young. Los Pantanos de Villa. Biología y Conservación. Museo de Historia Natural - UNMSM. Serie de Divulgación $N^{\circ}$ 11: 85 - 103.

Schmidt, G 1986. CRC Handbook of Tapeworm Identification. CRC Press, INC. Florida. 675 pp.

Suresh, K. 1952. Cestode Parasites of Birds. Indian Journal of Helminthology, 4: 1-72.

Tantaleán, M.; R. Martinez y D. Juárez. 1974/1975. Estudio de Algunos Tremátodos del Perú. Rev. Per. Med. Tropical. U.N.M.S.M. 3-4 (1): 46-56.

Tantaleán M.; L. Sarmiento y A. Huiza. 1992. Digeneos (Trematoda) del Perú. Boletín de Lima. $\mathrm{N}^{\circ} 11$. $47-84$. 